\title{
Artificial-Intelligence-Based Techniques to Evaluate Switching Overvoltages during Power System Restoration
}

\author{
Iman Sadeghkhani, ${ }^{1}$ Abbas Ketabi, ${ }^{2}$ and Rene Feuillet ${ }^{3}$ \\ ${ }^{1}$ Department of Electrical Engineering, Islamic Azad University, Najafabad Branch, Najafabad 85141-43131, Iran \\ ${ }^{2}$ Department of Electrical Engineering, University of Kashan, Kashan 87317-51167, Iran \\ ${ }^{3}$ Grenoble Electrical Engineering Lab (G2ELab), Grenoble INP, BP46, 38402 Saint Martin d'Hères Cedex, France \\ Correspondence should be addressed to Iman Sadeghkhani; i.sadeghkhani@ec.iut.ac.ir
}

Received 19 May 2012; Revised 31 July 2012; Accepted 23 October 2012

Academic Editor: Richard Mitchell

Copyright (C) 2013 Iman Sadeghkhani et al. This is an open access article distributed under the Creative Commons Attribution License, which permits unrestricted use, distribution, and reproduction in any medium, provided the original work is properly cited.

\begin{abstract}
This paper presents an approach to the study of switching overvoltages during power equipment energization. Switching action is one of the most important issues in the power system restoration schemes. This action may lead to overvoltages which can damage some equipment and delay power system restoration. In this work, switching overvoltages caused by power equipment energization are evaluated using artificial-neural-network- (ANN-) based approach. Both multilayer perceptron (MLP) trained with LevenbergMarquardt (LM) algorithm and radial basis function (RBF) structure have been analyzed. In the cases of transformer and shunt reactor energization, the worst case of switching angle and remanent flux has been considered to reduce the number of required simulations for training ANN. Also, for achieving good generalization capability for developed ANN, equivalent parameters of the network are used as ANN inputs. Developed ANN is tested for a partial of 39-bus New England test system, and results show the effectiveness of the proposed method to evaluate switching overvoltages.
\end{abstract}

\section{Introduction}

In recent years, due to economic competition and deregulation, power systems are being operated closer and closer to their limits. At the same time, power systems have increased in size and complexity. Both factors increase the risk of major power outages. After a blackout, power needs to be restored as quickly and reliably as possible, and, consequently, detailed restoration plans are nec [1-4].

One of the major concerns in power system restoration is the occurrence of overvoltages as a result of switching procedures. These can be classified as transient overvoltages, sustained overvoltages, harmonic resonance overvoltages, and overvoltages resulting from ferroresonance. Steady-state overvoltages occur at the receiving end of lightly loaded transmission lines as a consequence of line-charging currents (reactive power balance). Excessive sustained overvoltages may lead to damage of transformers and other power system equipment. Transient overvoltages are a consequence of switching operations on long transmission lines, or the switching of capacitive devices, and may result in arrester failures. Ferroresonance is a nonharmonic resonance characterized by overvoltages whose waveforms are highly distorted and can cause catastrophic equipment damages $[1,5]$.

Overvoltage will put the transformer into saturation, causing core heating and copious harmonic current generation. Circuit breaker called upon to operate during periods of high voltage will have reduced interrupting capability. At some voltage, even the ability to interrupt line-charging current will be lost [6-8].

This paper presents the artificial neural network (ANN) application for estimation of peak and duration of overvoltages under switching transients during transformer, shunt reactor, and transmission lines energization.

In [6], switching overvoltages during single-phase transformer have been evaluated using multilayer perceptron (MLP) trained with Levenberg-Marquardt (LM) algorithm. In this paper, three-phase transformer has been analyzed. 
Many time-domain simulations are required to train ANN resulting in a large amount of simulation time. For the transformer and shunt reactor energization study, this paper uses a harmonic index which can calculate overvoltages for the worst case of switching time and remanent flux [6]. This index reduces ANN training time effectively. Also, there is no need to specify switching time and remanent flux. A tool such as proposed in this paper that can give the maximum switching overvoltage and its duration will be helpful to the operator during system restoration. Also it can be used as a training tool for the operators. Developed ANN is tested for a partial of 39-bus New England test system to illustrate the proposed approach.

\section{Study System Modelling}

The electrical components of the network are modeled using the Power System Blockset (PSB) (MATLAB/Simulink-based simulation tool $[9,10])$. These models should be adapted for the desired frequency range (here the frequencies up to $f=10 f_{0}$ are considered to be sufficient). The generator is represented by an ideal voltage source behind the subtransient inductance in series with the armature winding resistance that can be as accurate as the Park model [11]. Phase of voltage source is determined by the load flow results. Transmission lines are described by distributed line models. The circuit breaker is represented by an ideal switch. The transformer model takes into account the winding resistances $\left(R_{1}, R_{2}\right)$, the leakage inductances $\left(L_{1}, L_{2}\right)$, and the magnetizing characteristics of the core, which is modeled by a resistance, $R_{m}$, simulating the core active losses and a saturable inductance, $L_{\text {sat }}$. The shunt reactor model takes into account the leakage inductance as well as the magnetizing characteristics of the core, which is modeled by a resistance, $R_{m}$, simulating the core active losses and a saturable inductance, $L_{\text {sat }}$. The saturation characteristic is specified as a piecewise linear characteristic [12]. All of the loads are modeled as constant impedances.

\section{Training Artificial Neural Network}

In this paper, two ANN structures have been used: multilayer perceptron (MLP) trained with Levenberg-Marquardt (LM) algorithm and radial basis function (RBF). Detailed structures of MLP-LM and RBF are presented in [13] and [14], respectively.

Percentage error is calculated as follows:

$$
\operatorname{error}(\%)=\frac{|\mathrm{ANN}-\mathrm{PSB}|}{\mathrm{PSB}} \times 100 \text {. }
$$

The sample system considered for explanation of the proposed methodology and ANN training is a $400 \mathrm{kV}$ extrahigh-voltage (EHV) network shown in Figure 1. The equivalent circuit parameters are added to ANN inputs to achieve good generalization capability for trained ANN. In fact, in this approach ANN is trained just once for sample system of Figure 1. Since ANN training is based on equivalent circuit parameters, developed ANN can be used for every studied

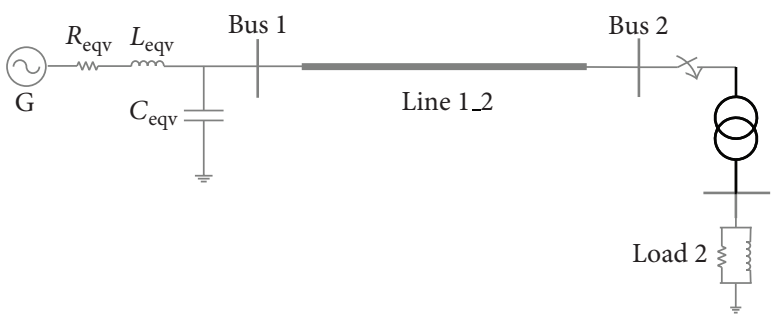

(a)

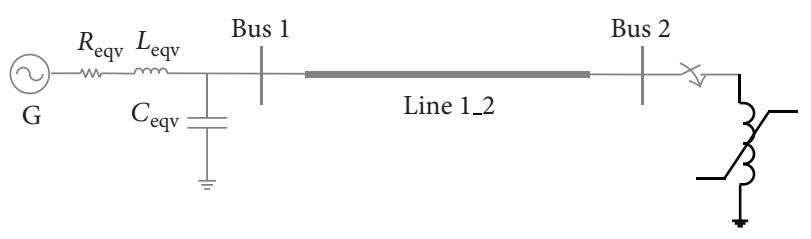

(b)

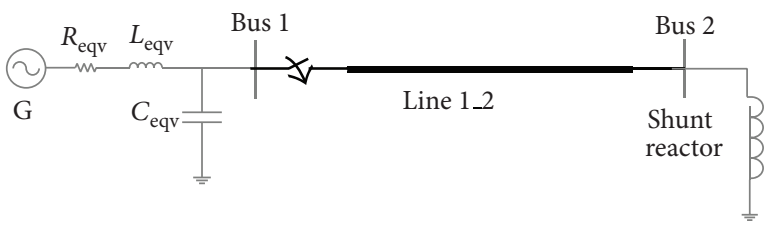

(c)

FIGURE 1: Sample systems for power components energization study. (a) Transformer energization, (b) shunt reactor energization, and (c) transmission line energization. G: generator, $R_{\text {eqv }}$ : equivalent resistance, $L_{\text {eqv }}$ : equivalent inductance, and $C_{\text {eqv }}$ : equivalent capacitance.

system. In other words, it is just sufficient to convert every studied system to the equivalent system of Figure 1, then it is possible to use developed ANN to estimate overvoltages.

\section{Transformer Energization Study}

The major cause of harmonic resonance overvoltage problems is the switching of lightly loaded transformers at the end of transmission lines. The harmonic-current components of the same frequency as the system resonance frequencies are amplified in case of parallel resonance, thereby creating higher voltages at the transformer terminals. This leads to a higher level of saturation, resulting in higher harmonic components of the inrush current that again results in increased voltages. This can happen particularly in lightly damped systems, common at the beginning of a restoration procedure when a path from a black-start source to a large power plant is being established and only a few loads are restored yet $[1,5]$.

Normally for harmonic overvoltages analysis during transformer energization, the worst case of the switching angle and remanent flux must be considered, which is a function of switching time, transformer characteristics and its initial flux condition, and impedance characteristics of the switching bus [12]. Using the worst switching angle and remanent flux, the number of simulations for each case can be reduced significantly. 
TABLE 1: Effect of switching time and remanent flux on the maximum of overvoltages and duration of $V_{\text {peak }}>1.3$ p.u.

\begin{tabular}{lccc}
\hline $\begin{array}{l}\text { Switching } \\
\text { angle (deg.) }\end{array}$ & $\begin{array}{l}\text { Remanent } \\
\text { flux (p.u.) }\end{array}$ & $\begin{array}{c}V_{\text {peak }} \\
\text { p.u. })\end{array}$ & $\begin{array}{c}\text { Duration of } \\
\left(V_{\text {peak }}>1.3 \text { p.u. }\right)(\mathrm{s})\end{array}$ \\
\hline \multicolumn{4}{c}{ Transformer study } \\
\hline 39 & 0.65 & 2.1961 & 0.7544 \\
87 & 0.62 & 1.8095 & 0.4627 \\
60 & 0.09 & 1.8831 & 0.8469 \\
15 & 0.42 & 2.0482 & 0.5134 \\
\hline \multicolumn{4}{c}{ Shunt reactor study } \\
20 & 0.27 & 1.9205 & 0.2753 \\
75 & 0.65 & 1.5841 & 0.5628 \\
60 & 0.27 & 1.6537 & 0.3394 \\
\hline
\end{tabular}

In order to determine the worst-case switching time and remanent flux, the following index is defined as

$$
W=\sum_{h=2}^{10} Z_{j j}(h) \cdot I_{j}\left(h, t_{0}, \phi_{r}\right),
$$

where $t_{0}$ is the switching time, $\phi_{r}$ is the initial transformer flux, and $h$ is the harmonic order. This index can be a definition for the worst-case switching condition and remanent flux. Using a numerical algorithm, one can find the switching time and remanent flux for which $W$ is maximal (i.e., harmonic overvoltages are maximal).

Figure 2(a) shows the result of the PSB frequency analysis at bus 2 in Figure 1(a). The magnitude of the Thévenin impedance, seen from bus $2, Z_{\text {bus } 2}$, shows a parallel resonance peak at $246 \mathrm{~Hz}$. Figures 2(b), 2(c), and 2(d) show changes of $W$ index with respect to the current starting angle and remanent flux for three phases. As shown in Figure 2, $W_{\max , B}$ is bigger than $W_{\max , A}$ and $W_{\max , C}$. Therefore, if simulation is performed based on switching angle and remanent flux related to $W_{\max , B}$, maximum overvoltages are achieved. Table 1 summarizes the results of overvoltages simulation for five different switching angles and remanent flux that includes $W_{\max , A}\left(80^{\circ}\right.$ and 0.62 p.u. $), W_{\max , B}\left(39^{\circ}\right.$ and 0.65 p.u.), and $W_{\max , C}\left(87^{\circ}\right.$ and 0.09 p.u.). Results verify the effectiveness of $W$ index.

As mentioned in [6], the most important aspects which influence the transformer overvoltages are voltage at transformer bus before switching, line length, switching angle, saturation curve slope, and remanent flux. This information will help the operator to select the proper sequence of transformer to be energized safely with transients appearing safe within the limits. As mentioned in a previous section, equivalent resistance, equivalent inductance, and equivalent capacitance have been added to ANN inputs to enhance generalization capability. Based on (2) and Figure 2, switching angle and remanent flux are omitted from ANN inputs; thus, ANN training procedure time is reduced significantly. Therefore, in this paper for training $\mathrm{ANN}$, the following parameters are considered as ANN inputs: (i) voltage at transformer bus before switching,

(ii) equivalent resistance of the network,

(iii) equivalent inductance of the network,

(iv) equivalent capacitance of the network,

(v) line length,

(vi) saturation curve slope.

The steps for harmonic overvoltages assessment and estimation are listed as follows:

(1) determine the characteristics of transformer that should be energized,

(2) calculate the $Z_{i i}(h)$ at the transformer bus for $h=$ $2 f_{0}, \ldots, 10 f_{0}$,

(3) compute the worst switching angle and remanent flux for simulation,

(4) run PSB simulation,

(5) determine the overvoltage peak and duration,

(6) repeat the steps 1 to 5 with various system parameters to learn artificial neural network,

(7) test the artificial neural network with different system parameters.

Schematic diagram of transformer energization study during power system restoration is illustrated in Figure 3.

\section{Study of Shunt Reactor Energization}

Long EHV transmission lines are generally compensated by means of shunt reactor sets. Reactor failures have directed attention to the transient overvoltages generated by reactor switching. Shunt reactors are applied to regulate the reactive power balance of a system by means of compensating for the surplus reactive power generation of transmission lines. Reactors are normally disconnected at heavy load and are connected to the lines at periods of low load. Consequently, frequent switching is a significant characteristic of shunt reactors in order that they can react to the changing system load condition [15].

Transients caused by shunt reactor switching have been an important parameter in the design of the relevant equipment (reactor, circuit breaker, and insulation) of power systems [16]. Based on considered model for shunt reactor, it is possible to use harmonic index which is defined in a previous section. Table 1 summarizes the results of overvoltages simulation for four different switching angles and remanent flux that verify the effectiveness of $W$ index for shunt reactor energization study.

The most important parameters which influence the shunt reactor overvoltages are voltage at shunt reactor bus before switching, line length, switching angle, saturation curve slope, remanent flux, and shunt reactor capacity. Using $W$ index, switching angle and remanent flux have been omitted from ANN inputs. Also, equivalent circuit parameters are added to ANN inputs to increase generalization capability. Consequently, in this case, the following parameters are selected as ANN inputs: 


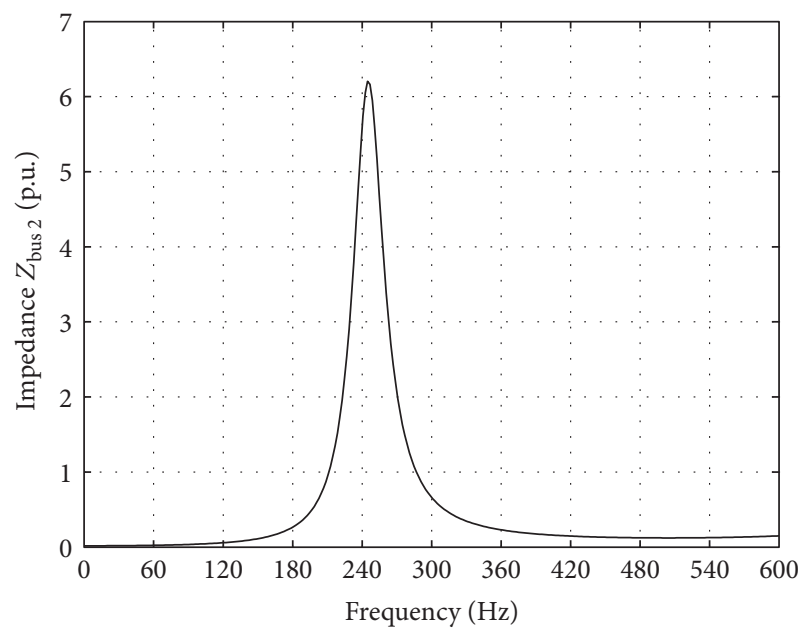

(a)

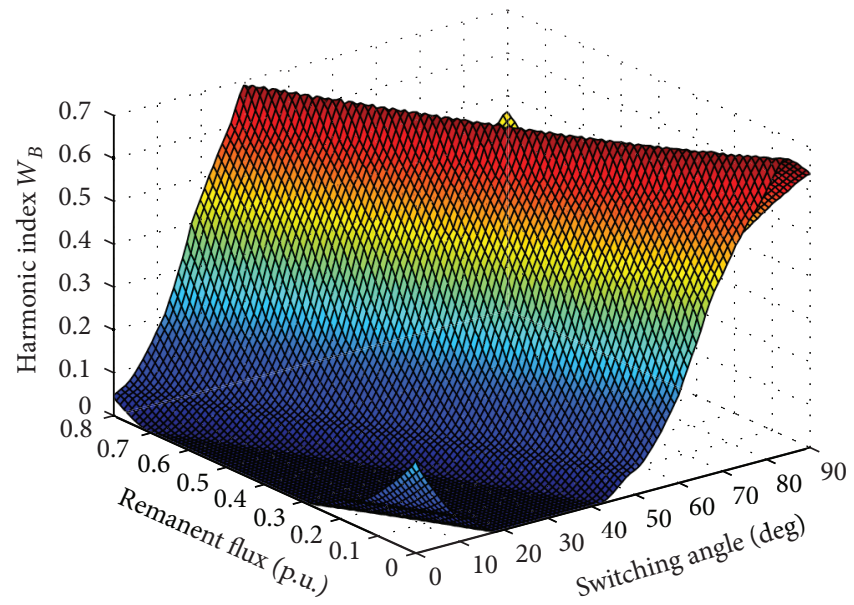

(c)

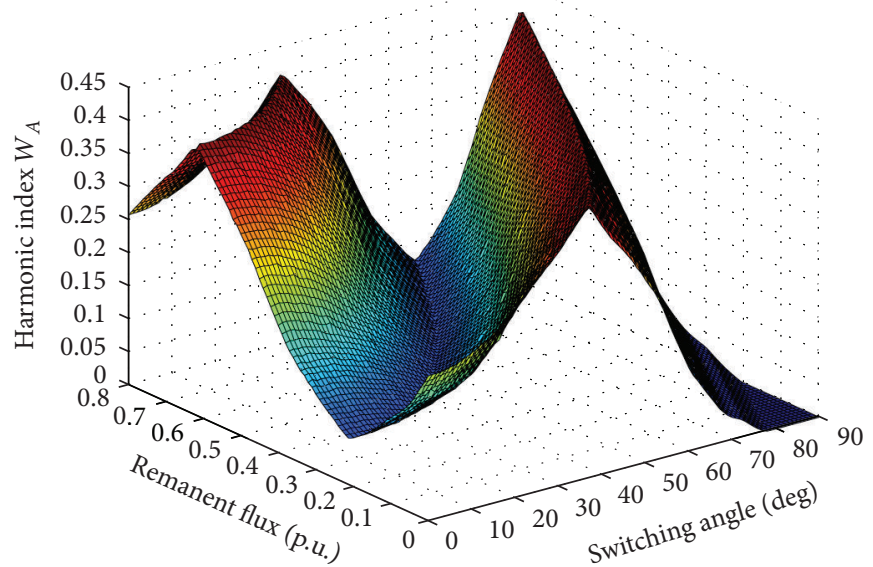

(b)

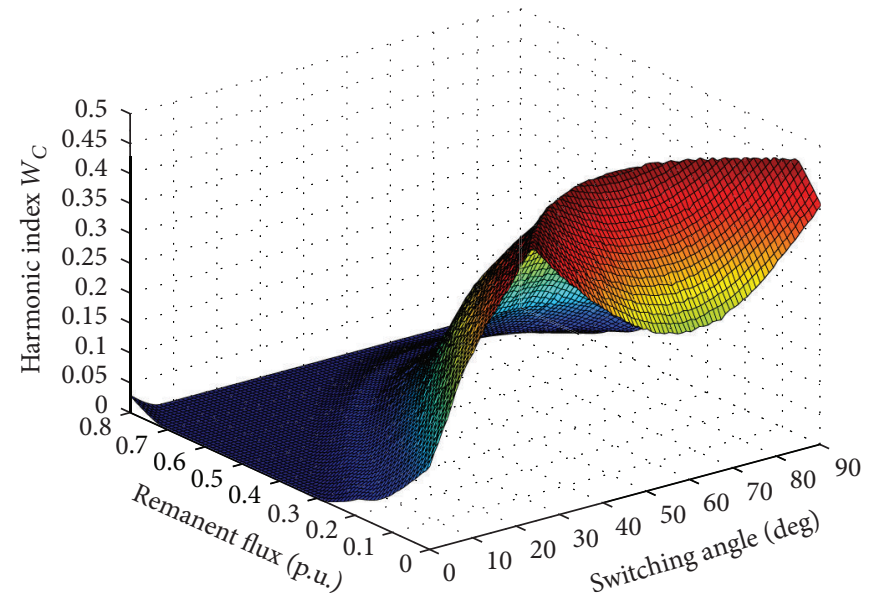

(d)

Figure 2: (a) Impedance at bus 2 and changes of $W$ index with respect to current starting angle and remanent flux in (b) phase A, (c) phase $\mathrm{B}$, and $(\mathrm{d})$ phase $\mathrm{C}$.

(i) voltage at shunt reactor bus before switching,

(ii) equivalent resistance of the network,

(iii) equivalent inductance of the network,

(iv) equivalent capacitance of the network,

(v) line length,

(vi) shunt reactor capacity,

(vii) saturation curve slope. follow:

The steps of overvoltages assessment and estimation

(1) determine the characteristics of shunt reactor that should be energized,

(2) calculate the $Z_{i i}(h)$ at the shunt reactor bus for $h=$ $2 f_{0}, \ldots, 10 f_{0}$,

(3) compute the worst switching angle and remanent flux for simulation,

(4) run PSB simulation,
(5) determine the overvoltage peak and duration,

(6) repeat the steps 1 to 5 with various system parameters to learn artificial neural network,

(7) test the artificial neural network with different system parameters.

\section{Transmission Line Energization Study}

In most countries, the main step in the process of power system restoration, following a complete/partial blackout, is energization of primary restorative transmission lines. Switching overvoltage is of a primary importance in insulation coordination for extra-high-voltage (EHV) lines. The objective of simulating switching overvoltage is to help for a proper insulation coordination and would lead to minimize damage and interruption to service as a consequence of steady-state, dynamic, and transient overvoltage [17].

During the early stages of restoring high-voltage overhead and underground transmission lines, concerns are with three 


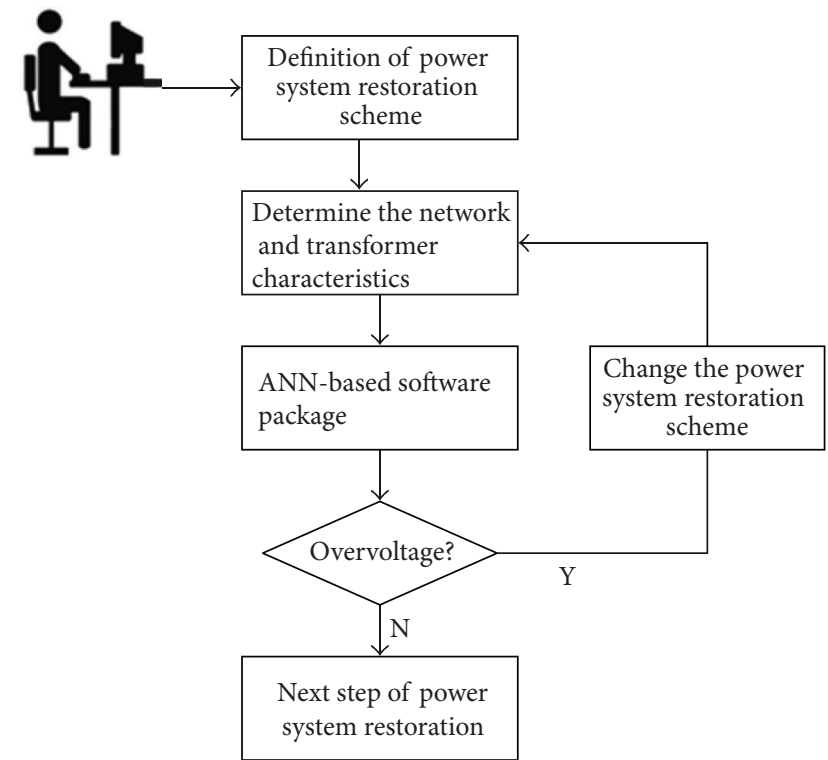

FigURE 3: ANN-based approach to analyze harmonic overvoltages during transformer energization.

related overvoltages: sustained power frequency overvoltages, switching transients (surges), and harmonic resonance. In the early stages of the restoration, the lines are lightly loaded; resonance therefore is lightly damped, which in turn means that the resulting resonance voltages may be very high [1].

In order to reduce the steady-state overvoltage of noload transmission lines, a shunt reactor is connected at the receiving end of transmission line.

In practical system, a number of factors affect the overvoltages factors due to energization or reclosing. The most important factors are voltage at sending-end bus of transmission line before switching, switching angle, line length, line capacitance, and shunt reactor capacity. Equivalent circuit parameters are added to ANN inputs to increase generalization capability. Consequently, in this case, following parameters are considered as ANN inputs:

(i) voltage at the sending-end bus of the transmission line before switching,

(ii) equivalent resistance of the network,

(iii) equivalent inductance of the network,

(iv) equivalent capacitance of the network,

(v) closing time of the circuit breaker poles,

(vi) line length,

(vii) line capacitance,

(viii) shunt reactor capacity.

The steps for transient overvoltages estimation are listed as follows:

(1) determine the characteristics of transmission line that should be energized,

(2) run PSB simulation,

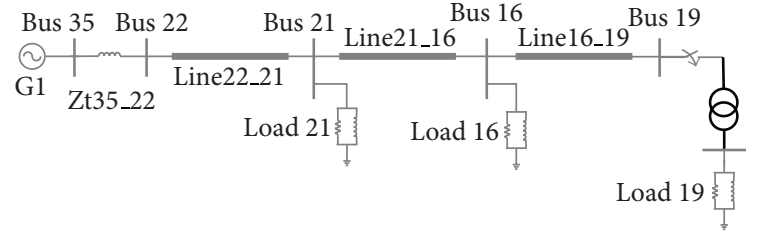

(a)

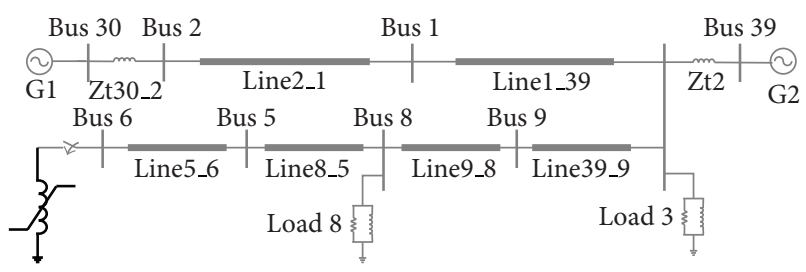

(b)

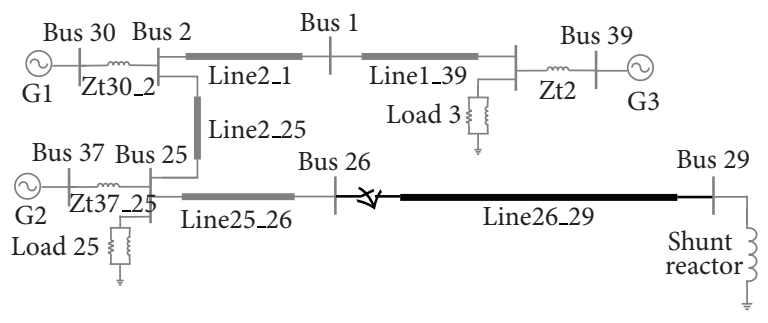

(c)

FIgure 4: Portions of 39-bus New England test systems: (a) transformer energization, (b) shunt reactor energization, and (c) transmission line energization.

(3) determine the overvoltage peak and duration,

(4) repeat the steps 1 to 3 with various system parameters to learn artificial neural network,

(5) test the artificial neural network with different system parameters.

\section{Case Study}

In this section, the proposed algorithm is demonstrated for three case studies that are a portion of 39-bus New England test system which is shown in Figure 4, and its parameters are listed in [18].

For training ANNs (three cases), all experiments have been repeated for different system parameters which form 2000 sets. For producing these sets, ANN inputs have been varied in proper range for each parameter. 1000 sets were used to train each ANN, and 1000 sets were used to test each ANN. Table 2 shows specifications of developed ANNs.

Figure 4(a) shows a one-line diagram of a portion of 39bus New England test system which is in restorative state. The generator at bus 35 is a black-start unit. The load 19 shows cranking power of the later generator that must be restored by the transformer of bus 19 . When the transformer is energized, harmonic overvoltages can be produced because the transformer is lightly loaded. The equivalent circuit of this system that is seen behind bus 16 is determined, and values of equivalent resistance, equivalent inductance, and 
TABLE 2: Specifications of trained ANNs.

\begin{tabular}{lccccc}
\hline & \multicolumn{2}{c}{ Number of hidden layers } & \multicolumn{2}{c}{ Number of neurons in hidden layers } & \multicolumn{2}{c}{ Training time (epochs) } \\
& LM & RBF & LM & RBF & LM \\
\hline Transformer energization & 2 & 2 & 8 & 10 & 45 \\
Shunt reactor energization & 2 & 2 & 8 & 10 & 72 \\
Transmission line energization & 2 & 2 & 7 & 9 & 61 \\
\hline
\end{tabular}

TABLE 3: Some sample testing data and output for LM algorithm.

\begin{tabular}{|c|c|c|c|c|c|c|c|c|c|}
\hline \multicolumn{10}{|c|}{ Transformer energization } \\
\hline$V$ & L.L. & & & $V_{\mathrm{PSB}}$ & $V_{\mathrm{LM}}$ & Error $_{V}$ & $T_{\mathrm{PSB}}$ & $T_{\mathrm{LM}}$ & Error $_{T}$ \\
\hline 0.9314 & 155 & & & 1.3252 & 1.3684 & 3.2576 & 0.1264 & 0.1237 & 2.1682 \\
\hline 0.9668 & 182 & & & 1.4577 & 1.4324 & 1.7386 & 0.3819 & 0.3644 & 4.5937 \\
\hline 0.9812 & 200 & & & 1.5031 & 1.4807 & 1.4928 & 0.3285 & 0.3378 & 2.8406 \\
\hline 1.0435 & 215 & & & 1.6602 & 1.6498 & 0.6239 & 0.5094 & 0.5143 & 0.9571 \\
\hline 1.0752 & 237 & & & 1.8535 & 1.9076 & 2.9175 & 0.6673 & 0.6438 & 3.5194 \\
\hline 1.1373 & 256 & & & 1.9892 & 1.9396 & 2.4916 & 0.5961 & 0.6039 & 1.3058 \\
\hline 1.1781 & 288 & & & 2.0545 & 2.0879 & 1.6271 & 0.7105 & 0.7197 & 1.2935 \\
\hline 1.2098 & 310 & & & 2.0361 & 2.0934 & 2.8132 & 0.9254 & 0.9457 & 2.1962 \\
\hline \multicolumn{10}{|c|}{ Shunt reactor energization } \\
\hline V & L.L. & S.R. & & $V_{\mathrm{PSB}}$ & $V_{\mathrm{LM}}$ & Error $_{V}$ & $T_{\mathrm{PSB}}$ & $T_{\mathrm{LM}}$ & Error $_{T}$ \\
\hline 1.1442 & 150 & 70 & & 1.5011 & 1.4497 & 3.4218 & 0.1936 & 0.1904 & 1.6459 \\
\hline 1.1561 & 165 & 45 & & 1.5453 & 1.5857 & 2.6149 & 0.2375 & 0.2351 & 1.0246 \\
\hline 1.2302 & 178 & 30 & & 1.6769 & 1.7241 & 2.8137 & 0.3469 & 0.3628 & 4.5728 \\
\hline 1.2514 & 200 & 30 & & 1.7481 & 1.7194 & 1.6424 & 0.3952 & 0.4043 & 2.3071 \\
\hline 1.3326 & 215 & 23 & & 1.9507 & 2.0117 & 3.1295 & 0.5104 & 0.5195 & 1.7915 \\
\hline 1.3326 & 215 & 17 & & 1.9914 & 1.9749 & 0.8261 & 0.5536 & 0.5571 & 0.6248 \\
\hline 1.4165 & 230 & 17 & & 2.1652 & 2.1759 & 0.4936 & 0.6742 & 0.6594 & 2.1963 \\
\hline 1.4327 & 242 & 10 & & 2.2479 & 2.2172 & 1.3674 & 0.7593 & 0.7395 & 2.6134 \\
\hline \multicolumn{10}{|c|}{ Transmission line energization } \\
\hline$V$ & L.L. & S.R. & S.A. & $V_{\mathrm{PSB}}$ & $V_{\mathrm{LM}}$ & Error $_{V}$ & $T_{\mathrm{PSB}}$ & $T_{\mathrm{LM}}$ & Error $_{T}$ \\
\hline 0.9491 & 375 & 40 & 30 & 2.3508 & 2.4024 & 2.1962 & 0.3652 & 0.3704 & 1.4328 \\
\hline 0.9127 & 240 & 40 & 30 & 2.2769 & 2.2328 & 1.9381 & 0.3107 & 0.3159 & 1.6649 \\
\hline 0.9973 & 240 & 55 & 60 & 2.3016 & 2.3849 & 3.6172 & 0.3496 & 0.3458 & 1.0793 \\
\hline 0.9754 & 195 & 12 & 75 & 2.3882 & 2.4523 & 2.6835 & 0.4073 & 0.4034 & 0.9458 \\
\hline 1.0719 & 315 & 23 & 15 & 2.4195 & 2.4071 & 0.5124 & 0.4217 & 0.4257 & 0.9426 \\
\hline 1.0592 & 282 & 45 & 5 & 2.3725 & 2.3413 & 1.3157 & 0.3846 & 0.3948 & 2.6419 \\
\hline 1.0946 & 137 & 63 & 45 & 2.3596 & 2.3902 & 1.2984 & 0.3378 & 0.3424 & 1.3674 \\
\hline 1.1123 & 346 & 10 & 53 & 2.8537 & 2.7944 & 2.0791 & 0.5449 & 0.5543 & 1.7204 \\
\hline
\end{tabular}

$V$ : voltage at power component bus before switching (p.u.), L.L.: line length (km), S.R.: shunt reactor capacity (MVAR), S.A.: switching angle (deg.), $V_{\mathrm{PSB}}$ : overvoltage peak calculated by PSB (p.u.), $V_{\mathrm{LM}}$ : overvoltage peak calculated by LM (p.u.), $T_{\mathrm{PSB}}$ : overvoltage duration calculated by PSB (s), $T_{\mathrm{LM}}$ : overvoltage duration calculated by LM (s), error ${ }_{V}$ : voltage error (\%), and error $_{T}$ : duration time error (\%).

equivalent capacitance are calculated. In other words, the case study system is converted to equivalent system as in Figure 1(a). Values of equivalent resistance, equivalent inductance, and equivalent capacitance are 0.00326 p.u., 0.02793 , and 1.8561 p.u., respectively. For testing trained ANN, values of voltage at transformer bus (bus 19) and line length are varied, and overvoltage peak and duration are calculated using developed ANN. Table 3 contains the same sample result of test data of transformer energization for LM algorithm, and Table 4 has these data for RBF structure.
As another example, the system in Figure 4(b) is examined. In the next step of the restoration, unit at bus 6 must be restarted. In order to reduce the steady-state overvoltage of no-load transmission lines, the reactor at bus 6 should be energized. In this condition, harmonic overvoltages can be produced. After calculating equivalent circuit seen from bus 5 , various cases of shunt reactor energization are taken into account, and corresponding overvoltages peak and duration are computed from PSB program and trained ANN. In this case, values of equivalent resistance, equivalent inductance, 
TABLE 4: Some sample testing data and output for RBF structure.

\begin{tabular}{|c|c|c|c|c|c|c|c|c|c|}
\hline \multicolumn{10}{|c|}{ Transformer energization } \\
\hline$V$ & L.L. & & & $V_{\mathrm{PSB}}$ & $V_{\mathrm{RBF}}$ & Error $_{V}$ & $T_{\mathrm{PSB}}$ & $T_{\mathrm{RBF}}$ & Error $_{T}$ \\
\hline 0.9314 & 155 & & & 1.3252 & 1.3496 & 1.8426 & 0.1264 & 0.1274 & 0.8255 \\
\hline 0.9668 & 182 & & & 1.4577 & 1.4526 & 0.3479 & 0.3819 & 0.3694 & 3.2697 \\
\hline 0.9812 & 200 & & & 1.5031 & 1.4759 & 1.8103 & 0.3285 & 0.3356 & 2.1762 \\
\hline 1.0435 & 215 & & & 1.6602 & 1.6156 & 2.6851 & 0.5094 & 0.5183 & 1.7531 \\
\hline 1.0752 & 237 & & & 1.8535 & 1.8772 & 1.2794 & 0.6673 & 0.6802 & 1.9357 \\
\hline 1.1373 & 256 & & & 1.9892 & 2.0191 & 1.5008 & 0.5961 & 0.5929 & 0.5374 \\
\hline 1.1781 & 288 & & & 2.0545 & 2.0150 & 1.9217 & 0.7105 & 0.6911 & 2.7264 \\
\hline 1.2098 & 310 & & & 2.0361 & 2.0791 & 2.1135 & 0.9254 & 0.9386 & 1.4215 \\
\hline \multicolumn{10}{|c|}{ Shunt reactor energization } \\
\hline$V$ & L.L. & S.R. & & $V_{\mathrm{PSB}}$ & $V_{\mathrm{RBF}}$ & Error $_{V}$ & $T_{\mathrm{PSB}}$ & $T_{\mathrm{RBF}}$ & Error $_{T}$ \\
\hline 1.1442 & 150 & 70 & & 1.5011 & 1.5268 & 1.7135 & 0.1936 & 0.1881 & 2.8214 \\
\hline 1.1561 & 165 & 45 & & 1.5453 & 1.5356 & 0.6281 & 0.2375 & 0.2268 & 4.5184 \\
\hline 1.2302 & 178 & 30 & & 1.6769 & 1.6141 & 3.7428 & 0.3469 & 0.3557 & 2.5369 \\
\hline 1.2514 & 200 & 30 & & 1.7481 & 1.7206 & 1.5746 & 0.3952 & 0.3902 & 1.2657 \\
\hline 1.3326 & 215 & 23 & & 1.9507 & 1.9955 & 2.2974 & 0.5104 & 0.5214 & 2.1624 \\
\hline 1.3326 & 215 & 17 & & 1.9914 & 1.9295 & 3.1108 & 0.5536 & 0.5612 & 1.3751 \\
\hline 1.4165 & 230 & 17 & & 2.1652 & 2.2251 & 2.7659 & 0.6742 & 0.6407 & 4.9752 \\
\hline 1.4327 & 242 & 10 & & 2.2479 & 2.2811 & 1.4782 & 0.7593 & 0.7809 & 2.8395 \\
\hline \multicolumn{10}{|c|}{ Transmission line energization } \\
\hline$V$ & L.L. & S.R. & S.A. & $V_{\mathrm{PSB}}$ & $V_{\mathrm{RBF}}$ & Error $_{V}$ & $T_{\mathrm{PSB}}$ & $T_{\mathrm{RBF}}$ & Error $_{T}$ \\
\hline 0.9491 & 375 & 40 & 30 & 2.3508 & 2.3126 & 1.6245 & 0.3652 & 0.3748 & 2.6184 \\
\hline 0.9127 & 240 & 40 & 30 & 2.2769 & 2.3064 & 1.2951 & 0.3107 & 0.3051 & 1.7963 \\
\hline 0.9973 & 240 & 55 & 60 & 2.3016 & 2.3363 & 1.5084 & 0.3496 & 0.3421 & 2.1507 \\
\hline 0.9754 & 195 & 12 & 75 & 2.3882 & 2.3757 & 0.5219 & 0.4073 & 0.4126 & 1.2985 \\
\hline 1.0719 & 315 & 23 & 15 & 2.4195 & 2.3328 & 3.5826 & 0.4217 & 0.4297 & 1.9046 \\
\hline 1.0592 & 282 & 45 & 5 & 2.3725 & 2.3924 & 0.8397 & 0.3846 & 0.3691 & 4.0293 \\
\hline 1.0946 & 137 & 63 & 45 & 2.3596 & 2.2892 & 2.9841 & 0.3378 & 0.3306 & 2.1266 \\
\hline 1.1123 & 346 & 10 & 53 & 2.8537 & 2.8927 & 1.3682 & 0.5449 & 0.5399 & 0.9157 \\
\hline
\end{tabular}

$V$ : voltage at power component bus before switching (p.u.), L.L.: line length (km), S.R.: shunt reactor capacity (MVAR), S.A.: switching angle (deg.), $V_{\text {PSB }}$ : overvoltage peak calculated by PSB (p.u.), $V_{\mathrm{RBF}}$ : overvoltage peak calculated by RBF (p.u.), $T_{\mathrm{PSB}}$ : overvoltage duration calculated by PSB (s), $T_{\mathrm{RBF}}$ : overvoltage duration calculated by RBF (s), error $V$ : voltage error $(\%)$, and error ${ }_{T}$ : duration time error (\%).

and equivalent capacitance are 0.00577 p.u., 0.02069 , and 0.99 p.u., respectively. The summary of few results is presented in Table 3 for LM algorithm and Table 4 for RBF structure.

For testing developed ANN for transmission lines energization study, the system in Figure 4(c) is examined, that is, another portion of 39-bus New England test system. In the next step of the restoration, line 26_29 must be restarted. As mentioned before, first this system is converted to equivalent circuit of Figure 1(c). In this case, values of equivalent resistance, equivalent inductance, and equivalent capacitance are 0.00792 p.u., 0.0247 , and 1.1594 p.u., respectively. For testing developed ANN, various cases of transmission line energization are taken into account, and corresponding peak and duration of overvoltages are computed from PSB program and trained ANN. The summary of few results is presented in Table 3 for LM algorithm and Table 4 for RBF structure. It can be seen from the results that the LM algorithm and RBF structure are able to estimate overvoltage peak and duration with good accuracy. Maximum voltage and duration time error for LM algorithm are $3.6172 \%$ and $4.5937 \%$, respectively. These errors are proper for power systems because the acceptable range for voltage variation is $\pm 5 \%$. Also, maximum voltage and duration time error for RBF structure are $3.7428 \%$ and $4.9752 \%$, respectively, which are within the acceptable range.

\section{Conclusion}

This paper introduced an ANN-based method to evaluate switching overvoltages during power equipment energization. Both MLP-LM and RBF structures have been used to train ANN. To achieve good generalization capability for developed ANN, its training is performed using equivalent circuit parameters of the network. For transformer and shunt reactor studies, a harmonic index has been used which evaluates switching overvoltages for the worst case of switching 
time and remanent flux. This index reduces training time of ANN effectively. Also, there is no need to specify switching time and remanent flux. The results from both LM and RBF schemes are close to results from the conventional method and can assist prediction of the overvoltage of other case studies within the range of training set. Simulation results for a partial 39-bus New England test system show that the proposed method can evaluate switching overvoltages properly, and it can be used as an operator-training tool during power system restoration.

\section{References}

[1] M. M. Adibi, R. W. Alexander, and B. Avramovic, "Overvoltage control during restoration," IEEE Transactions on Power Systems, vol. 7, no. 4, pp. 1464-1470, 1992.

[2] A. Ketabi, A. M. Ranjbar, and R. Feuillet, "Analysis and control of temporary overvoltages for automated restoration planning," IEEE Transactions on Power Delivery, vol. 17, no. 4, pp. 1121-1127, 2002.

[3] S. A. Taher and I. Sadeghkhani, "Estimation of magnitude and time duration of temporary overvoltages using ANN in transmission lines during power system restoration," Simulation Modelling Practice and Theory, vol. 18, no. 6, pp. 787-805, 2010.

[4] A. Ketabi, I. Sadeghkhani, and R. Feuillet, "Using artificial neural network to analyze harmonic overvoltages during power system restoration," European Transactions on Electrical Power, vol. 21, no. 7, pp. 1941-1953, 2011.

[5] G. Morin, "Service restoration following a major failure on the hydro-quebec power system," IEEE Transactions on Power Delivery, vol. 2, no. 2, pp. 454-463, 1987.

[6] A. Ketabi, I. Sadeghkhani, and R. Feuillet, "Using artificial neural network to analyze harmonic overvoltages during power system restoration," European Transactions on Electrical Power, vol. 21, no. 7, pp. 1941-1953, 2011.

[7] D. Thukaram, H. P. Khincha, and S. Khandelwal, "Estimation of switching transient peak overvoltages during transmission line energization using artificial neural network," Electric Power Systems Research, vol. 76, no. 4, pp. 259-269, 2006.

[8] I. Sadeghkhani, A. Ketabi, and R. Feuillet, "Radial basis function neural network application to power system restoration studies," Computational Intelligence and Neuroscience, vol. 2012, Article ID 654895, 2012.

[9] G. Sybille, P. Brunelle, L. Hoang, L. A. Dessaint, and K. AlHaddad, "Theory and applications of power system blockset, a MATLAB/Simulink-based simulation tool for power systems," in Proceedings of IEEE Power Engineering Society Winter Meeting, pp. 774-779, 2000.

[10] A. Ketabi and I. Sadeghkhani, Electric Power Systems Simulation Using MATLAB, Morsal Publications, 2011.

[11] M. M. Duró, "Damping modelling in transformer energization studies for system restoration: some standard models compared to field measurements," in Proceedings of IEEE Bucharest PowerTech: Innovative Ideas Toward the Electrical Grid of the Future, Bucharest, Romania, July 2009.

[12] G. Sybille, M. M. Gavrilovic, J. Belanger, and V. Q. Do, "Transformer saturation effects on EHV system overvoltages," IEEE Transactions on Power Apparatus and Systems, vol. 104, no. 3, pp. 671-680, 1985.
[13] M. T. Hagan and M. B. Menhaj, "Training feedforward networks with the Marquardt algorithm," IEEE Transactions on Neural Networks, vol. 5, no. 6, pp. 989-993, 1994.

[14] A. Karami and M. S. Mohammadi, "Radial basis function neural network for power system load-flow," International Journal of Electrical Power and Energy Systems, vol. 30, no. 1, pp. 60-66, 2008.

[15] L. Prikler, G. Bán, and G. Bánfai, "EMTP models for simulation of shunt reactor switching transients," International Journal of Electrical Power and Energy Systems, vol. 19, no. 4, pp. 235-240, 1997.

[16] I. Sadeghkhani, A. Ketabi, and R. Feuillet, "Radial basis function neural network application to measurement and control of shunt reactor overvoltages based on analytical rules," Mathematical Problems in Engineering, vol. 2012, Article ID 647305, 2012.

[17] T. Keokhoungning, S. Premrudeepreechacharn, and K. Ngamsanroaj, "Evaluation of switching overvoltage in $500 \mathrm{kV}$ transmission line interconnection Nam Theun 2 power plant to Roi Et 2 substation," in Proceedings of the Asia-Pacific Power and Energy Engineering Conference (APPEEC '09), March 2009.

[18] S. Wunderlich, M. M. Adibi, R. Fischl, and C. O. D. Nwankpa, "Approach to standing phase angle reduction," IEEE Transactions on Power Systems, vol. 9, no. 1, pp. 470-478, 1994. 

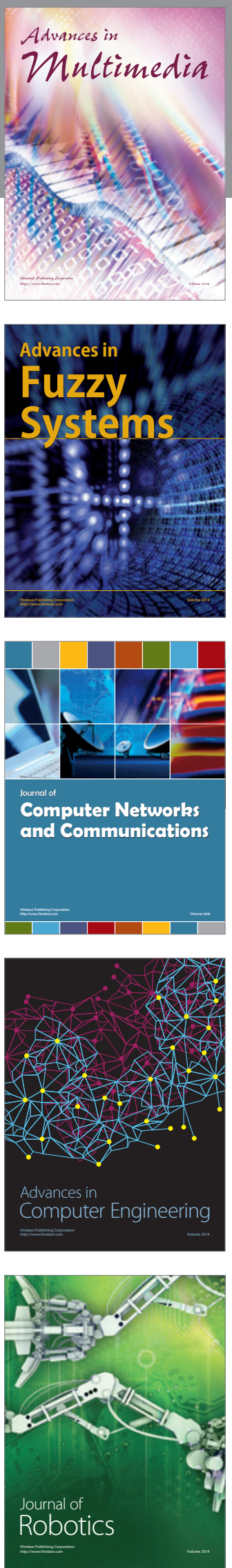

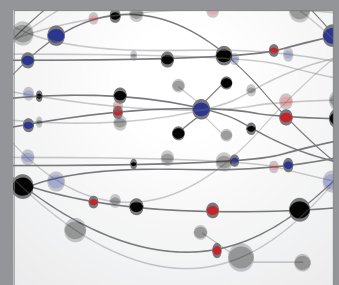

The Scientific World Journal
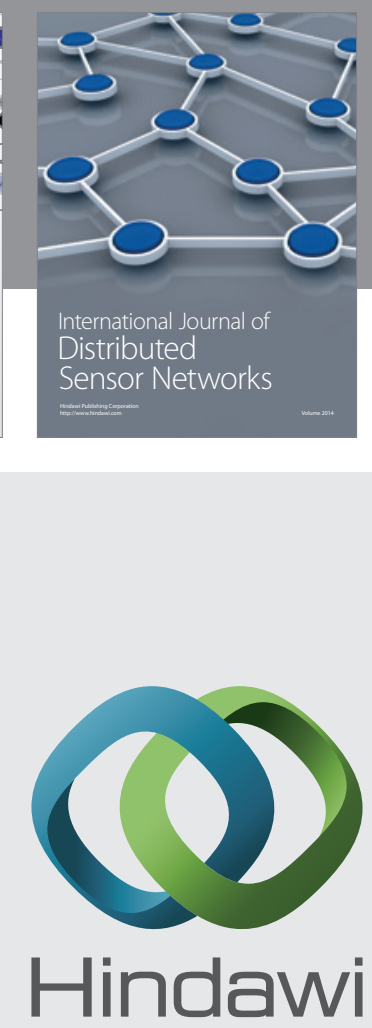

Submit your manuscripts at

http://www.hindawi.com
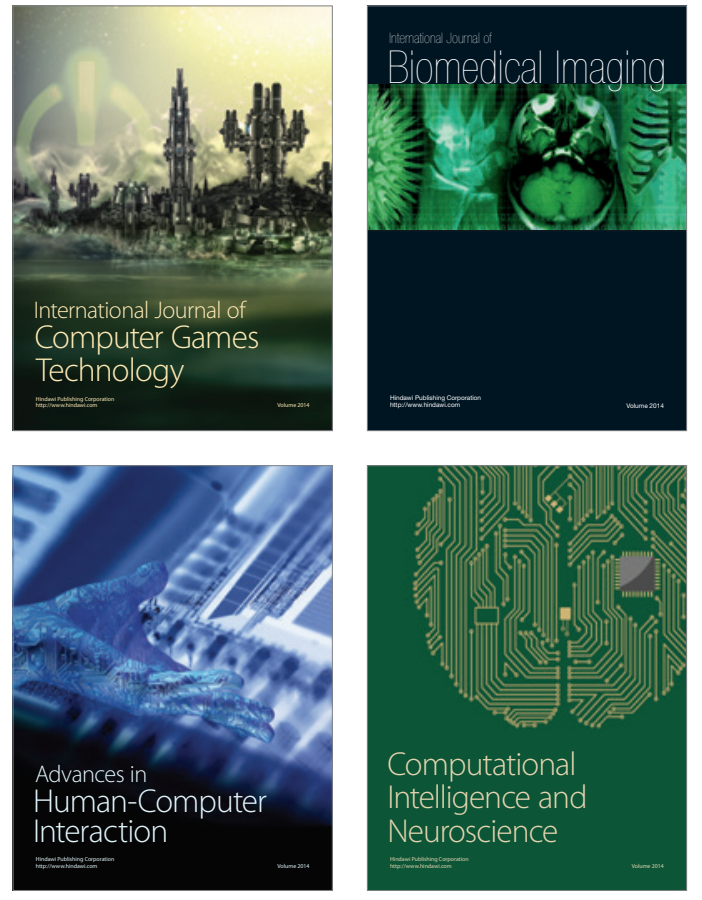
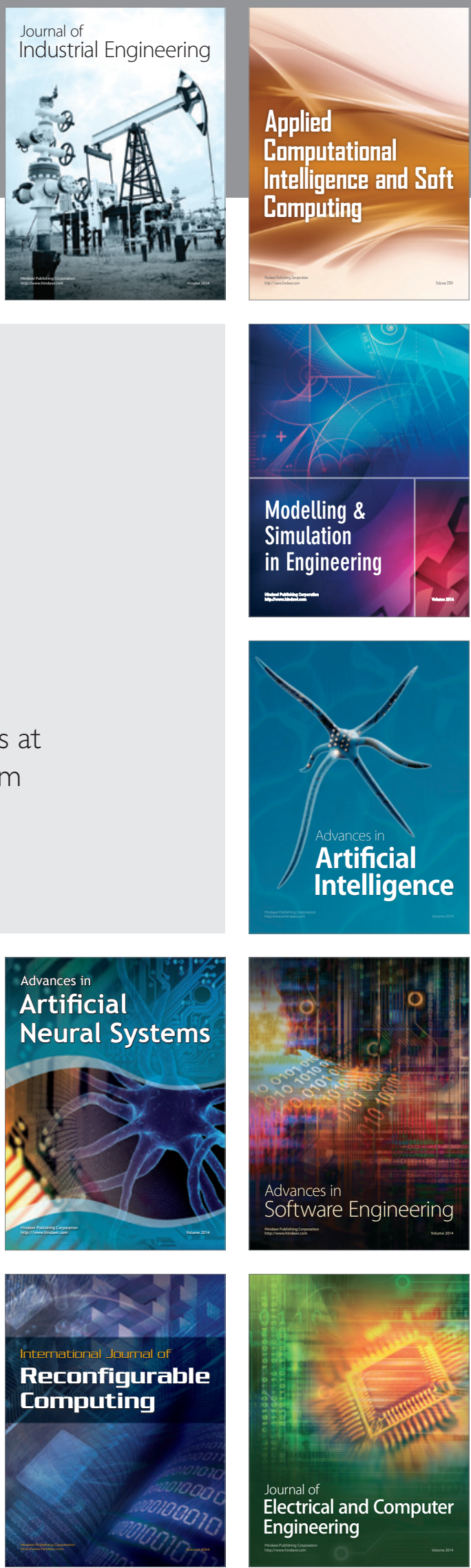